\title{
An Inverse Problem for the Helmholtz Equation Involving Two Semi-Infinite Fluids
}

\author{
A. Chakrabarti ${ }^{1, *}$ Prabir Daripa ${ }^{2, \dagger}$ and S. Roy ${ }^{3}$ \\ ${ }^{1}$ Department of Mathematics, NJIT, University Heights, Newark, NJ-07102 \\ ${ }^{2}$ Department of Mathematics, Texas A\&M University, College Station, TX-77843 \\ ${ }^{3}$ Department of Mathematics, IIT-Madras, Chennai-600036, India
}

\begin{abstract}
An analytical method is developed for solving an inverse problem for Helmholtz's equation associated with two semi-infinite incompressible fluids of different variable refractive indices, separated by a plane interface. The unknowns of the inverse problem are: (i) the refractive indices of the two fluids, (ii) the ratio of the densities of the two fluids, and (iii) the strength of an acoustic source assumed to be situated at the interface of the two fluids. These are determined from the pressure on the interface produced by the acoustic source. The effect of the surface tension force at the interface is taken into account in this paper.

The application of the proposed analytical method to solve the inverse problem is also illustrated with several examples. In particular, exact solutions of two direct problems are first derived using standard classical methods which are then used in
\end{abstract}

\footnotetext{
*Permanent Address: Indian Institute of Science, Bangalore - 560012, India

$\dagger$ Author for correspondence, (e-mail: prabir.daripa@math.tamu.edu)
} 
our proposed inverse method to recover the unknowns of the corresponding inverse problems. The results are found to be in excellent agreement.

\section{Introduction}

The inverse problem concerning the determination of the triple $\left\{n(z), \alpha, h_{0}\right\}$, arising in acoustics, is considered where the three unknowns (the triple) of the problem are as described below:

(i) $n(z)$, the variable refraction coefficient of the medium (see section 2 below) consisting of two semi-infinite fluids of constant densities $\rho_{0+}$ and $\rho_{0-}$ occupying the regions $z>0$ and $z<0$ respectively, where the $z$-axis is taken normal to the interface and a rectangular coordinate system $(x, y, z)$ is employed.

(ii) $\alpha=\frac{\rho_{0-}}{\rho_{0+}}>1$.

(iii) $h_{0}$, a constant, determining the strength of a time-harmonic $\left(e^{-i \omega t}\right)$ sound-source of creating fluid assumed to be situated at $z=0^{+}$, very near the origin of coordinates on the interface $z=0$.

The known data of the problem under consideration are the acoustic pressure functions $p\left(x, y, 0^{ \pm}\right)$at the interface where $p\left(x, y, 0^{ \pm}\right)=\lim _{z \rightarrow 0^{ \pm}} p(x, y, z)$. These are discussed in detail in section 2 below.

Similar inverse problems have been considered for solution by Ramm [1] among other authors using a Green's function approach when the effect of surface tension at the interface of the two fluids is neglected. The present paper deals with the above inverse problem when the effect of the surface tension at the interface of the two fluids is also taken into account. Below we briefly summarize the method of solving this inverse problem which is given in more detail in the main body of the paper. 
In the present context, the corresponding direct problem is concerned with the determination of the pressure functions in the fluid regions. It is assumed in our approach below that this direct problem possesses a unique solution for the class of functions $n(z)$, having at most an algebraic growth at infinity. Under this assumption, the direct problem is cast into an integral equation of the second kind (see Mikhlin [2]) for the determination of the pressure functions. Using a technique for obtaining a series solution of this integral equation for the pressure functions which is valid for sufficiently small values of the wave number $k=\frac{\omega}{c_{0}}\left(c_{0}\right.$ representing the constant sound speed in free-space), the inverse problem of determination of $n(z)$ is cast into two independent Laplace-inversion problems, one for each of the two regions: $z>0$ and $z<0$. The other two unknowns $\alpha$ and $h_{0}$ (in the triple $\left.\left\{n(z), \alpha, h_{0}\right\}\right)$ are determined by using a straight-forward limiting procedure.

The paper is organized as follows. In section 2, we have presented the basic equations and boundary conditions of the direct problem. Section 3 is devoted to the detailed analysis to determine the solution of the inverse problem. For validation purposes of our inverse method, two special cases of the direct problem are considered which are as follows: (i) $n(z)=n_{0}$ for $z>0$, and $n(z)=n_{1}$ for $z<0$ where $n_{0}$ and $n_{1}$ are two different known constants; and (ii) $n(z)=n_{0}$ (a known constant) for $z>0$, and $n(z)=\exp (2 z)$ for $z<0$. These two direct problems are solved in a straight-forward manner to obtain the input data for the inverse problem. With these as the input data, the results of the corresponding inverse problems for the unknowns are found to be in excellent agreement with their exact values when the various formulae derived in section 3 for the solution of the inverse problems are utilized.

\section{Mathematical Formulation}

We employ the notations used by Jones [3] with $p_{0}$ and $\rho_{0}$ as constant pressure and density respectively of a single fluid medium and with $p_{0}(1+p)$ and $\rho_{0}(1+\rho)$ as the corresponding perturbed quantities, caused due to a sound source of creating fluid and situated at a point 
$\left(x_{0}, y_{0}, z_{0}\right)$. Then the basic equations of acoustics can be written down in the following linearized form (neglecting gravity, viscosity and thermal conductivity, and assuming that $p$ and the perturbed fluid velocity $\mathbf{v}$ are small quantities):

$$
\begin{aligned}
& \rho_{0} \frac{\partial \rho}{\partial t}+\rho_{0} \operatorname{div} \mathbf{v}=-\frac{\partial m}{\partial t} \delta\left(x-x_{0}\right) \delta\left(y-y_{0}\right) \delta\left(z-z_{0}\right), \\
& \rho_{0} \frac{\partial \mathbf{v}}{\partial t}=-p_{0} \operatorname{grad} p \\
& p=c^{2} \frac{\rho_{0} \rho}{p_{0}}
\end{aligned}
$$

where ' $c$ ' is the speed of sound and $m(t)$ represents the mass created at the source at time $t, \delta(x)$ denoting Dirac's delta function (considering isentropic flows only).

If the medium under consideration consists of two semi-infinite fluids of constant densities $\rho_{0+}$ and $\rho_{0-}$ occupying the regions $z>0$ and $z<0$ respectively, and if the time-dependence is assumed to be of the type $\exp (-i \omega t)$ (i.e. time-harmonic case), the above equations are to be used with appropriate changes in the various quantities involved. In addition to this, we assume that the sound speed $c(z)$ varies in the $z$-direction only. The source of sound is taken to be of the form: $m(t)=m_{0} \exp (-i \omega t),\left(m_{0}\right.$ denoting the strength of the source) located at $\left(x_{0}, y_{0}, z_{0}\right)=(0,0, a), a(>0)$. Then, by means of a standard elimination procedure adapted to the Eqs. (1), (2), and (3), we obtain the following partial differential equation for the pressure-function $p(x, y, z)$.

$$
\left(\frac{\partial^{2}}{\partial x^{2}}+\frac{\partial^{2}}{\partial y^{2}}+\frac{\partial^{2}}{\partial z^{2}}+k^{2} n(z)\right) p=-h_{0} k^{2} \delta(x) \delta(y) \delta(z-a)
$$

where $n(z)=c_{0}^{2} / c^{2}(z), k=\omega / c_{0}$, and $h_{0}=m_{0} c_{0}^{2} / p_{0^{+}}$, with $p_{0^{+}}$representing the constant unperturbed pressure in the region $z>0$, and $c_{0}$ representing the constant sound speed in free-space (or any reference medium). The function $n(z)$, related to the refractive index $c_{0} / c(z)$ of the fluid medium under consideration, will be called the refraction coefficient of the medium. It is worth pointing out that the functions $n(z)$ and $p$, appearing in Eq. (4), actually represent two different functions in the two regions $z>0$ and $z<0$ and that there are two equations built-in in Eq. (4). 
The kinematic and dynamic interface conditions (see Drazin [4], Lamb [5]) are respectively the following.

(a) The particle-displacement at the interface $z=0$ must be continuous, and

(b) The jump in normal stress (pressure) at the interface must be balanced with the surfacetension force $T$ at the interface.

The linearized versions of these interface conditions can be expressed (See Lamb [5] for details) in the following forms:

$$
\begin{aligned}
\alpha \frac{\partial p_{+}}{\partial z} & =\frac{\partial p_{-}}{\partial z}, \quad \text { on } \quad z=0, \\
p_{+}-p_{-} & =\frac{-\varepsilon}{k^{2}}\left(\frac{\partial^{2}}{\partial x^{2}}+\frac{\partial^{2}}{\partial y^{2}}\right) \frac{\partial p_{+}}{\partial z}, \quad \text { on } \quad z=0,
\end{aligned}
$$

where $\alpha=\rho_{0-} / \rho_{0+}$, and $\varepsilon=T / c_{0}^{2} \rho_{0+}$ are two parameters of the problem. Here, and in the sequel, we have used the symbols $p_{+}$and $p_{-}$for the pressure function $p$ in the two regions $z>0$ and $z<0$ respectively.

In addition to the above interface conditions, the functions $p_{+}$and $p_{-}$must also satisfy the radiation conditions at infinity in order to ensure the uniqueness of the solution of the boundary value problem posed by the Eqs. (4)-(6). We assume that the unknown function $n(z)$ tends to a constant limit as $|z| \rightarrow \infty$ so that the radiation conditions are applicable in the two regions $z>0$ and $z<0$.

This concludes the formulation of the direct problem of acoustics where $n(z), \alpha, h_{0}, \varepsilon, a$ and $k$ are known and the pressure function is unknown. But, what we wish to consider here is the inverse problem of determining the triple $\left\{n(z), \alpha, h_{0}\right\}$ from a knowledge of the data $p_{+}$and $p_{-}$at the interface $z=0$ when $\varepsilon, a$, and $k$ are given. In the next section, we have presented an analytical method of solution of this inverse problem under the assumption that $a \rightarrow 0^{+}$, i.e., the source is placed at the interface. 


\section{Method of Solution}

We first non-dimensionalize the partial differential equation (4) and the conditions (5) and (6) by using the non-dimensional coordinates $\bar{x}, \bar{y}, \bar{z}$ and the non-dimensional parameters $\bar{k}, \bar{\varepsilon}$, and $\bar{h}_{0}$ as defined by relations

$$
\bar{x}=x / L, \quad \bar{y}=y / L, \quad \bar{z}=z / L, \quad \bar{k}=k L, \quad \bar{\varepsilon}=\varepsilon / L, \quad \bar{h}_{0}=h_{0} / L^{3} \quad \text { and } \quad \bar{a}=a / L,
$$

$L$ representing a typical length-scale. We then drop the bars everywhere and proceed to solve the problem as described below.

Using a double Fourier transform as defined by

$$
\tilde{p}(z)=\int_{-\infty}^{\infty} \int_{-\infty}^{\infty} p(x, y, z) \exp \left[i\left(\lambda_{1} x+\lambda_{2} y\right)\right] d x d y, \quad\left(\lambda^{2}=\lambda_{1}^{2}+\lambda_{2}^{2}\right)
$$

the boundary value problem posed by the Eqs. (4)-(6) can be reduced to the problem of solving the following integral equation (see [6],[7]): (using the assumption that $n(z)$ possesses at most an algebraic growth as $|z| \rightarrow \infty)$

$$
\tilde{p}\left(z^{\prime}\right)=k^{2} h_{0} G\left(a, z^{\prime}\right)+k^{2} \int_{-\infty}^{\infty} n(z) G\left(z, z^{\prime}\right) \tilde{p}(z) d z, \quad\left(-\infty<z^{\prime}<\infty\right)
$$

where the function $G\left(z, z^{\prime}\right)$ satisfies the following equation and conditions:

$$
\begin{aligned}
& \left(\frac{\partial^{2}}{\partial z^{2}}-\lambda^{2}\right) G=-\delta\left(z-z^{\prime}\right), \\
& \frac{\partial G_{+}}{\partial z}=\frac{\partial G_{-}}{\partial z}, \quad G_{+}=\alpha G_{-}+\beta \frac{\partial G_{-}}{\partial z}, \quad \text { on } \quad z=0, \\
& G\left(z, z^{\prime}\right) \rightarrow 0 \quad \text { as } \quad|z| \rightarrow \infty
\end{aligned}
$$

with $\beta=\varepsilon \lambda^{2} / k^{2}$. Here $G_{+}=G\left(z, z^{\prime}\right)$ for $z>0$, and $G_{-}=G\left(z, z^{\prime}\right)$ for $z<0$.

The exact determination of the function $G\left(z, z^{\prime}\right)$ can be carried out in a straight-forward fashion and we find that 


\section{For $z^{\prime}>0$ :}

$$
G\left(z, z^{\prime}\right)= \begin{cases}\frac{1}{2 \lambda}\left[\exp \left(-\lambda\left|z-z^{\prime}\right|\right)+\frac{(\alpha+\beta \lambda-1)}{(\alpha+\beta \lambda+1)} \exp \left[-\lambda\left(z+z^{\prime}\right)\right]\right], & z>0 \\ \frac{1}{2 \lambda}\left[\frac{2}{(1+\alpha+\beta \lambda)} \exp \left[\lambda\left(z-z^{\prime}\right)\right]\right], & z<0\end{cases}
$$

II. For $z^{\prime}<0$ :

$$
G\left(z, z^{\prime}\right)= \begin{cases}\frac{1}{2 \lambda}\left[\frac{2 \alpha}{(1+\alpha+\beta \lambda)} \exp \left[\lambda\left(z^{\prime}-z\right)\right]\right], & z>0 \\ \frac{1}{2 \lambda}\left[\exp \left(-\lambda\left|z-z^{\prime}\right|\right)+\frac{(1-\alpha+\beta \lambda)}{(1+\alpha+\beta \lambda)} \exp \left[\lambda\left(z+z^{\prime}\right)\right]\right], & z<0 .\end{cases}
$$

Using these two forms of the function $G\left(z, z^{\prime}\right)$ in the above integral equation (8), we obtain

\section{For $z^{\prime}>0$,}

$$
\begin{aligned}
\tilde{p}\left(z^{\prime}\right)= & \frac{k^{2} h_{0}}{2 \lambda}\left[\exp \left(-\lambda\left|a-z^{\prime}\right|\right)+\left\{\frac{\varepsilon \lambda^{3}+(\alpha-1) k^{2}}{\varepsilon \lambda^{3}+(\alpha+1) k^{2}}\right\} \exp \left[-\lambda\left(a+z^{\prime}\right)\right]\right] \\
& +\frac{k^{4}}{\lambda\left[\varepsilon \lambda^{3}+(\alpha+1) k^{2}\right]} \int_{-\infty}^{0} n(z) \tilde{p}(z) \exp \left[\lambda\left(z-z^{\prime}\right)\right] d z \\
& +\frac{k^{2}}{2 \lambda} \int_{0}^{\infty} n(z) \tilde{p}(z)\left\{\exp \left(-\lambda\left|z-z^{\prime}\right|\right)+\left(\frac{\varepsilon \lambda^{3}+(\alpha-1) k^{2}}{\varepsilon \lambda^{3}+(\alpha+1) k^{2}}\right) \exp \left[-\lambda\left(z+z^{\prime}\right)\right]\right\} d z
\end{aligned}
$$

II. For $z^{\prime}<0$,

$$
\begin{aligned}
\tilde{p}\left(z^{\prime}\right)= & \frac{\alpha k^{4} h_{0}}{\lambda\left[\varepsilon \lambda^{3}+(\alpha+1) k^{2}\right]} \exp \left[\lambda\left(z^{\prime}-a\right)\right] \\
& +\frac{k^{2}}{2 \lambda} \int_{-\infty}^{0} n(z) \tilde{p}(z)\left\{\exp \left(-\lambda\left|z-z^{\prime}\right|\right)+\left(\frac{\varepsilon \lambda^{3}+(1-\alpha) k^{2}}{\varepsilon \lambda^{3}+(1+\alpha) k^{2}}\right) \exp \left[\lambda\left(z+z^{\prime}\right)\right]\right\} d z \\
& +\frac{\alpha k^{4}}{\lambda\left[\varepsilon \lambda^{3}+(\alpha+1) k^{2}\right]} \int_{0}^{\infty} n(z) \tilde{p}(z) \exp \left[\lambda\left(z^{\prime}-z\right)\right] d z
\end{aligned}
$$

We find that for the class of functions $n(z)$ under consideration (i.e., of algebraic growth at infinity), the integral equation for $\tilde{p}(z)$ as given by the Eqs. (14) and (15) possesses a unique 
solution for sufficiently small values of the parameter $k$. This establishes the existence of a unique solution of the direct problem for such small values of $k$. We write the solution of the Eqs. (14) and (15) in the form

$$
\tilde{p}(z)=k^{2} \tilde{p}_{2}(z)+k^{4} \tilde{p}_{4}(z)+k^{6} \tilde{p}_{6}(z)+\cdots,
$$

which is the equivalent to the Neumann series solution of the above integral equations.

In the special case, when $\varepsilon=0$, i.e., when the effect of surface tension is negligible, we find, from the relations (14) and (15), that all the terms of the expansion (16), except the first term, which contains the function $\tilde{p}_{2}$, are zero. In the case when $\varepsilon \neq 0$, we proceed to determine these functions in a manner as described below.

We multiply both sides of Eqs. (14) and (15) by the quantity $\lambda\left[\varepsilon \lambda^{3}+(\alpha+1) k^{2}\right]$ and substitute the relation (16). Then equating the terms involving $k^{2}, k^{4}$ and $k^{6}$, we arrive at the following results.

\section{(A) For $z^{\prime}>0$,}

$$
\begin{aligned}
\lambda \tilde{p}_{2}\left(z^{\prime}\right)= & \left(h_{0} / 2\right)\left[\exp \left(-\lambda\left|a-z^{\prime}\right|\right)+\exp \left[-\lambda\left(a+z^{\prime}\right)\right]\right], \\
\varepsilon \lambda^{4} \tilde{p}_{4}\left(z^{\prime}\right)+\lambda(1+\alpha) \tilde{p}_{2}\left(z^{\prime}\right)= & \left(h_{0} / 2\right)\left[(\alpha+1) \exp \left(-\lambda\left|a-z^{\prime}\right|\right)+(\alpha-1) \exp \left[-\lambda\left(a+z^{\prime}\right)\right]\right] \\
& +\left(\varepsilon \lambda^{3} / 2\right) \int_{0}^{\infty} n(z) \tilde{p}_{2}(z)\left\{\exp \left(-\lambda\left|z-z^{\prime}\right|\right)+\exp \left[-\lambda\left(z+z^{\prime}\right)\right]\right\} d z .
\end{aligned}
$$

\section{(B) For $z^{\prime}<0$,}

$$
\begin{aligned}
& \tilde{p}_{2}\left(z^{\prime}\right)=0 \\
& \varepsilon \lambda^{4} \tilde{p}_{4}\left(z^{\prime}\right)=\alpha h_{0} \exp \left[\lambda\left(z^{\prime}-a\right)\right] \\
& \varepsilon \lambda^{4} \tilde{p}_{6}\left(z^{\prime}\right)+\lambda(\alpha+1) \tilde{p}_{4}\left(z^{\prime}\right)=\left(\varepsilon \lambda^{3} / 2\right) \int_{-\infty}^{0} n(z) \tilde{p}_{4}(z) \exp \left\{-\lambda\left|z-z^{\prime}\right|\right]+\exp \left[\lambda\left(z+z^{\prime}\right)\right\} d z \\
& \quad+\alpha \int_{0}^{\infty} n(z) \tilde{p}_{2}(z) \exp \left[\lambda\left(z^{\prime}-z\right)\right] d z
\end{aligned}
$$


If we now take limits $z^{\prime} \rightarrow 0^{+}$and $z^{\prime} \rightarrow 0^{-}$of Eqs. (17b) and (18c) respectively, we obtain

$$
\begin{aligned}
& \int_{0}^{\infty} n(z) \exp (-\lambda z)\{\exp (-\lambda|a-z|)+\exp [-\lambda(a+z)]\} d z=\phi(\lambda), \\
& \int_{-\infty}^{0} n(z) \exp (2 \lambda z) d z=\psi(\lambda),
\end{aligned}
$$

where

$$
\begin{aligned}
& \phi(\lambda)=\frac{2}{\varepsilon \lambda^{2} h_{0}}\left[\varepsilon \lambda^{4} \tilde{p}_{4}\left(0^{+}\right)+h_{0} \exp (-\lambda a)\right], \\
& \psi(\lambda)=\frac{\lambda \exp (\lambda a)}{\alpha h_{0}}\left[\varepsilon \lambda^{4} \tilde{p}_{6}\left(0^{-}\right)+\frac{\alpha h_{0}}{\lambda}\left\{\frac{(\alpha+1) \exp (-\lambda a)}{\varepsilon \lambda^{2}}-\frac{1}{2} \phi(\lambda)\right\}\right] .
\end{aligned}
$$

It is easy to check from the above results that the interface conditions (5) and (6) are satisfied by the various components of the $\tilde{p}$-functions when series (16) for $\tilde{p}$ is substituted in these interface conditions.

While the results (19) and (20) can be viewed as two identities which must be satisfied by the refraction coefficients $n(z)$ of two fluid media under consideration, we can also utilize these results for the determination of the function $n(z)$ if the limiting values $\tilde{p}_{4}\left(0^{+}\right)$and $\tilde{p}_{6}\left(0^{-}\right)$are known (or equivalently, if $\tilde{p}\left(0^{+}\right)$and $\tilde{p}\left(0^{-}\right)$are known as shown below in equation (27)) with the assumption that $a \rightarrow 0^{+}$. Under such circumstances, we then solve the problem of determination of the function $n(z)$ by a Laplace inversion procedure.

In the results (19)-(22), if we take limit as $a \rightarrow 0^{+}$, we obtain

$$
\begin{gathered}
\int_{0}^{\infty} n(z) \exp (-2 \lambda z) d z=\frac{\phi_{0}(\lambda)}{2}, \\
\int_{-\infty}^{0} n(z) \exp (2 \lambda z) d z=\psi_{0}(\lambda)
\end{gathered}
$$

where

$$
\begin{aligned}
& \phi_{0}(\lambda)=\frac{2}{\varepsilon \lambda^{2} h_{0}}\left[\varepsilon \lambda^{4} \tilde{p}_{4}\left(0^{+}\right)+h_{0}\right] \\
& \psi_{0}(\lambda)=\frac{\lambda}{\alpha h_{0}}\left[\varepsilon \lambda^{4} \tilde{p}_{6}\left(0^{-}\right)+\frac{\alpha h_{0}}{\lambda}\left\{\frac{(\alpha+1)}{\varepsilon \lambda^{2}}-\frac{1}{2} \phi_{0}(\lambda)\right\}\right] .
\end{aligned}
$$


The results (23) and (24) can be viewed as Laplace transforms of the function $n(z)$ for $z>0$ and $z<0$ respectively. Hence the function $n(z)$ can be recovered by inverting these Laplace transforms (see [1]). As is well-known, these Laplace inversions require that the functions $\phi_{0}(\lambda)$ and $\psi_{0}(\lambda)$ are known for values of $\lambda$ lying on certain straight lines parallel to the imaginary axis (or equivalent to it), in the complex $\lambda$ plane. Hence the Laplace inversion problems given by the relations (25) and (26) are ill-posed problems and these can be resolved numerically by using the ideas proposed by Ramm ( see [9]).

It is important to recognize that all the functions appearing in the right of the relations (25) and (26) can be determined, from the knowledge of the given data $\tilde{p}\left(0^{ \pm}\right)$, which are the Fourier transforms of the limiting values of the pressure functions. In fact, by using the first results of the equations Eqs. (17a) and (18a) (when $a \rightarrow 0^{+}$), we obtain:

$$
\begin{aligned}
& \tilde{p}_{4}\left(0^{+}\right)=\lim _{k \rightarrow 0}\left\{\left[\tilde{p}\left(0^{+}\right)-k^{2} \tilde{p}_{2}\left(0^{+}\right)\right] k^{-4}\right\}=\lim _{k \rightarrow 0}\left\{\left[\tilde{p}\left(0^{+}\right)-k^{2} \lambda^{-1} h_{0}\right] k^{-4}\right\}, \\
& \tilde{p}_{6}\left(0^{-}\right)=\lim _{k \rightarrow 0}\left\{\left[\tilde{p}\left(0^{-}\right)-k^{4} \tilde{p}_{4}\left(0^{-}\right)\right] k^{-6}\right\}=\lim _{k \rightarrow 0}\left\{\left[\tilde{p}\left(0^{-}\right)-\frac{\alpha}{\varepsilon} k^{4} \lambda^{-4} h_{0}\right] k^{-6}\right\},
\end{aligned}
$$

so that the actual evaluation of the functions $\phi_{0}(\lambda)$ and $\psi_{0}(\lambda)$ can be carried out directly using the known data $\tilde{p}\left(0^{ \pm}\right)$.

The above procedure of recovering the unknown function $n(z)$ can be successfully completed, if the other two unknowns of the problem, i.e., the quantities $\alpha$ and $h_{0}$ are determined uniquely. To achieve this goal, we use Eqs. (17a) and (18b) and obtain the following formulae (when $a \rightarrow 0^{+}$).

$$
h_{0}=\lambda \tilde{p}_{2}\left(0^{+}\right), \quad \text { and } \quad \frac{\alpha h_{0}}{\varepsilon}=\lambda^{4} \tilde{p}_{4}\left(0^{-}\right) .
$$

Thus both the unknowns $h_{0}$ and $\alpha$ can be uniquely determined for a given data $\tilde{p}\left(0^{ \pm}\right)$and the relations that

$$
\tilde{p}_{2}\left(0^{+}\right)=\lim _{k \rightarrow 0}\left\{\tilde{p}\left(0^{+}\right) k^{-2}\right\}, \quad \text { and } \quad \tilde{p}_{4}\left(0^{-}\right)=\lim _{k \rightarrow 0}\left\{\tilde{p}\left(0^{-}\right) k^{-4}\right\}
$$

The inverse problem under consideration thus gets settled and its unique solution can be determined once the Laplace inversions of the two relations (23) and (24) are completed. 
The various formulae derived above can be used to construct the following algorithm to recover the values of the three unknowns $\left\{n(z), \alpha, h_{0}\right\}$ from input data $\tilde{p}\left(0^{ \pm}\right)$, provided such input data is either known a priori as a solution of the direct problem or obtained by solving a direct problem in a different manner for known values of the triple $\left\{n(z), \alpha, h_{0}\right\}$.

Inverse Algorithm-I: To recover $\left\{n(z), \alpha, h_{0}\right\}$ from $\tilde{p}\left(0^{ \pm}\right)$

[Note: Input data: $\tilde{p}\left(0^{ \pm}\right)$when $a \rightarrow 0^{+}$.]

Step 1. Find $\tilde{p}_{2}\left(0^{+}\right)$and $\tilde{p}_{4}\left(0^{-}\right)$from known input data $\tilde{p}\left(0^{ \pm}\right)$using $(29)$.

Step 2. Find $h_{0}$ and $\alpha$ using (28).

Step 3. Find $\tilde{p}_{4}\left(0^{+}\right)$and $\tilde{p}_{6}\left(0^{-}\right)$using $(27)$.

Step 4. Find $\Phi_{0}(\lambda)$ and $\Psi_{0}(\lambda)$ using (25) and (26) respectively.

Step 5. Finally, find $n(z)$, the refraction coefficient of the medium, by taking inverse Laplace transforms of $\Phi_{0}(\lambda)$ and $\Psi_{0}(\lambda)$ according to the relations (23) and (24).

We finally make the following important observations on the effectiveness of the above algorithm.

In steps 1 and 3 of the algorithm, it is required to calculate certain limits (Eqs. (29) and (27)) using the input data $p\left(x, y, 0^{\mp}\right)$. The problem of calculating these limits is equivalent to finding derivatives of the input data $p\left(x, y, 0^{\mp}\right)$ with respect to wavenumber $k$. Hence these derivatives must be well defined which in essence requires that the input data and its Fourier transform must be analytic functions of the wavenumber $k$.

In step 5 of the above algorithm, the Laplace inversions corresponding to integral equations (23) and (24) are to be performed numerically (which we have not taken up in this paper) by solving two moment problems (see Martin and Ramm [9]), which are the following integral equations of the first kind

$$
\int_{0}^{1} x^{m} n_{1}(x) d x=\frac{1}{2} \phi_{0}\left(\frac{m+1}{2}\right),
$$


and

$$
\int_{0}^{1} x^{m} n_{2}(x) d x=\psi_{0}\left(\frac{m+1}{2}\right),
$$

for $m=0,1,2,3, \ldots \ldots$ These are obtained by setting $x=\exp (-z), n_{1}(x)=n(-\ln x)$ for $z>0$ in Eq. (23), and $x=\exp (z), n_{2}(x)=n(\ln x)$ for $z<0$ in Eq. (24). The above algorithm is based on the existence of unique solution of the above two integral equations.

\section{Applications}

In order to illustrate the proposed method of solution of the inverse problem, by the procedure described above, we consider two special examples of the direct problem as described below. The known data for the corresponding inverse problems can be considered to be the ones obtained from the solutions of the direct problems which are determined by the standard techniques.

\subsection{Solutions to the direct problems}

Example - 1: Here the refractive index is chosen to be the following piecewise constant function.

$$
\left.\begin{array}{rl}
n(z) & =n_{0}, \quad z<0 \\
& =n_{1}, \quad z>0
\end{array}\right\}
$$

where $n_{0}, n_{1}$ are constants: $\alpha$ and $h_{0}$ are also assumed to be known constants.

We find that the solution of this direct problem can be written down immediately, satisfying the partial differential equation (4) and the boundary conditions (5) and (6) as given 
by the relations:

$$
\begin{aligned}
\tilde{p}(z)= & B_{1} \exp \left[-\left(\lambda^{2}-k^{2} n_{1}\right)^{1 / 2} z\right]+\frac{1}{2} k^{2} h_{0}\left(\lambda^{2}-k^{2} n_{1}\right)^{-1 / 2} \\
& \times\left[\exp \left\{\left(\lambda^{2}-k^{2} n_{1}\right)^{1 / 2}(z-a)\right\} H(a-z)\right. \\
& \left.+\exp \left\{-\left(\lambda^{2}-k^{2} n_{1}\right)^{1 / 2}(z-a)\right\} H(z-a)\right], \text { for } z>0, \\
\tilde{p}(z)= & C_{1} \exp \left[\left(\lambda^{2}-k^{2} n_{0}\right)^{1 / 2} z\right], \text { for } z<0,
\end{aligned}
$$

where

$$
\begin{aligned}
B_{1}= & \frac{1}{2} k^{2} h_{0} \exp \left[-\left(\lambda^{2}-k^{2} n_{1}\right)^{1 / 2} a\right]\left(\lambda^{2}-k^{2} n_{1}\right)^{-1 / 2} \\
& \times\left[\frac{\alpha k^{2}\left(\lambda^{2}-k^{2} n_{1}\right)^{1 / 2}-k^{2}\left(\lambda^{2}-k^{2} n_{0}\right)^{1 / 2}+\varepsilon \lambda^{2}\left(\lambda^{2}-k^{2} n_{0}\right)^{1 / 2}\left(\lambda^{2}-k^{2} n_{1}\right)^{1 / 2}}{\alpha k^{2}\left(\lambda^{2}-k^{2} n_{1}\right)^{1 / 2}+k^{2}\left(\lambda^{2}-k^{2} n_{0}\right)^{1 / 2}+\varepsilon \lambda^{2}\left(\lambda^{2}-k^{2} n_{0}\right)^{1 / 2}\left(\lambda^{2}-k^{2} n_{1}\right)^{1 / 2}}\right], \\
C_{1}= & {\left[\frac{\alpha h_{0} k^{4} \exp \left[-\left(\lambda^{2}-k^{2} n_{1}\right)^{1 / 2} a\right]}{\alpha k^{2}\left(\lambda^{2}-k^{2} n_{1}\right)^{1 / 2}+k^{2}\left(\lambda^{2}-k^{2} n_{0}\right)^{1 / 2}+\varepsilon \lambda^{2}\left(\lambda^{2}-k^{2} n_{0}\right)^{1 / 2}\left(\lambda^{2}-k^{2} n_{1}\right)^{1 / 2}}\right], }
\end{aligned}
$$

with $H(x)$ denoting the Heaviside function. Here $\tilde{p}(z)$ is the double Fourier transform of $p(x, y, z)$ as defined by the relation (7) [assuming that $\left(\lambda^{2}-k^{2} n_{0}\right)^{1 / 2}$ and $\left(\lambda^{2}-k^{2} n_{1}\right)^{1 / 2}$ both possess a positive real part, (this choice is required by the condition of vanishing of the transformed pressure function $\tilde{p}(z)$ as $|z| \rightarrow \infty$ (see, for instance, [7]))].

Example - 2: Here the refractive index is chosen to be

$$
\left.\begin{array}{rlr}
n(z) & =n_{0} & z>0 \\
& =\exp (2 z) & z<0
\end{array}\right\}
$$

where $n_{0}$ is a constant, $\alpha$ and $h_{0}$ are also assumed to be known constants as in the first example.

The differential equation for the transformed pressure function $\tilde{p}(z)$ for the region $z<0$ can be easily converted to a Bessel's differential equation by a standard transformation (see [8]). Using this idea, we find that the solution of the direct problem, which satisfies the partial differential equation (4) and the boundary conditions (5) and (6), can be expressed 
in the following form:

$$
\begin{aligned}
\tilde{p}(z)= & B_{2} \exp \left[-\left(\lambda^{2}-k^{2} n_{0}\right)^{1 / 2} z\right]+\frac{1}{2} k^{2} h_{0}\left(\lambda^{2}-k^{2} n_{0}\right)^{-1 / 2} \\
& \times\left[\exp \left\{\left(\lambda^{2}-k^{2} n_{0}\right)^{1 / 2}(z-a)\right\} H(a-z)\right. \\
& \left.+\exp \left\{-\left(\lambda^{2}-k^{2} n_{0}\right)^{1 / 2}(z-a)\right\} H(z-a)\right], \quad \text { for } z>0 \\
\tilde{p}(z)= & C_{2} \sum_{n=0}^{\infty} \frac{(-1)^{n}(k / 2)^{2 n+\lambda} \exp [(2 n+\lambda) z]}{n ! \Gamma(n+\lambda+1)}, \quad \text { for } z<0
\end{aligned}
$$

where

$$
\begin{aligned}
B_{2}= & \frac{1}{2} k^{2} h_{0} \exp \left[-\left(\lambda^{2}-k^{2} n_{0}\right)^{1 / 2} a\right]\left(\lambda^{2}-k^{2} n_{0}\right)^{-1 / 2} \\
& \times\left[\frac{\alpha k^{2}\left(\lambda^{2}-k^{2} n_{0}\right)^{1 / 2}-\left[k^{2}-\varepsilon \lambda^{2}\left(\lambda^{2}-k^{2} n_{0}\right)^{1 / 2}\right] \frac{J_{\lambda}^{\prime}(k)}{J_{\lambda}(k)}}{\left.\alpha k^{2}\left(\lambda^{2}-k^{2} n_{0}\right)^{1 / 2}+\left[k^{2}+\varepsilon \lambda^{2}\left(\lambda^{2}-k^{2} n_{0}\right)^{1 / 2}\right] \frac{J_{\lambda}^{\prime}(k)}{J_{\lambda}(k)}\right],}\right. \\
C_{2}= & {\left[\frac{\alpha h_{0} k^{4} \exp \left[-\left(\lambda^{2}-k^{2} n_{0}\right)^{1 / 2} a\right]}{J_{\lambda}(k)\left\{\alpha k^{2}\left(\lambda^{2}-k^{2} n_{0}\right)^{1 / 2}+\left[k^{2}+\varepsilon \lambda^{2}\left(\lambda^{2}-k^{2} n_{0}\right)^{1 / 2}\right] \frac{J_{\lambda}^{\prime}(k)}{J_{\lambda}(k)}\right\}}\right], }
\end{aligned}
$$

$J_{\lambda}(k)$ denoting the Bessel function of the first kind, and prime denoting derivative with respect to the argument.

\subsection{Solutions to the inverse problems}

The quantities $\tilde{p}\left(0^{ \pm}\right)$for the above two examples are determined from (33) and (35) respectively. The above Algorithm-I is then applied to recover the triple unknowns $\left\{n(z), \alpha, h_{0}\right\}$ for each of these two examples from corresponding known pressure data $p\left(x, y, 0^{ \pm}\right)$or equivalently Fourier transformed data $\tilde{p}\left(0^{ \pm}\right)$. We find that the recovered function $n(z)$ from the application of the above inverse algorithm agrees completely with the choices (32) and (34) for the two examples respectively. The other two unknown constants $\alpha$ and $h_{0}$ are also recovered completely. Some results obtained at various steps of the Algorithm-I when $a \rightarrow 0^{+}$ are given below. 


\section{For example 1:}

$$
\begin{aligned}
\tilde{p}_{2}\left(0^{+}\right) & =\frac{h_{0}}{\lambda} \\
\tilde{p}_{4}\left(0^{+}\right) & =\frac{h_{0}}{\lambda^{3}}\left[\frac{n_{1}}{2}-\frac{1}{\varepsilon \lambda}\right], \\
\tilde{p}_{4}\left(0^{-}\right) & =\frac{\alpha h_{0}}{\varepsilon \lambda^{4}}, \\
\tilde{p}_{6}\left(0^{-}\right) & =\frac{\alpha h_{0}}{\varepsilon \lambda^{6}}\left[-\frac{(\alpha+1)}{\varepsilon \lambda}+\frac{\left(n_{0}+n_{1}\right)}{2}\right], \\
\phi_{0}(\lambda) & =\frac{n_{1}}{\lambda} \\
\psi_{0}(\lambda) & =\frac{n_{0}}{2 \lambda} .
\end{aligned}
$$

II. For example 2:

$$
\begin{aligned}
\tilde{p}_{2}\left(0^{+}\right) & =\frac{h_{0}}{\lambda} \\
\tilde{p}_{4}\left(0^{+}\right) & =\frac{h_{0}\left(\varepsilon n_{0} \lambda-2\right)}{2 \varepsilon \lambda^{4}}, \\
\tilde{p}_{4}\left(0^{-}\right) & =\frac{\alpha h_{0}}{\varepsilon \lambda^{4}}, \\
\tilde{p}_{6}\left(0^{-}\right) & =\frac{\alpha h_{0}}{\varepsilon \lambda^{4}}\left[-\frac{(\alpha+1)}{\varepsilon \lambda^{3}}+\frac{n_{0}}{2 \lambda^{2}}+\frac{1}{2 \lambda(\lambda+1)}\right], \\
\phi_{0}(\lambda) & =\frac{n_{0}}{\lambda}, \\
\psi_{0}(\lambda) & =\frac{1}{2(\lambda+1)} .
\end{aligned}
$$

It is easily verified that original $n(z)$ for each of the two examples (see (32) and (34)) is recovered from taking the inverse Laplace transforms of $\phi_{0}(\lambda)$ and $\psi_{0}(\lambda)$ obtained above for both the examples. Alternatively, it can be seen that the $\phi_{0}(\lambda)$ and $\psi_{0}(\lambda)$ obtained above are consistent with (23) and (24) for each of the choices for $n(z)$ (see (32) and (34)) in the two examples. We have thus verified our proposed method of solution of the inverse problem through two examples. 


\section{Acknowledgments}

It is a pleasure to thank the referees for their suggestions.

\section{References}

[1] A.G. Ramm, Inverse Problems, An inverse problem for the Helmholtz equation in a semi-infinite medium, 3 (1987), L19.

[2] S.G. Mikhlin, Integral Equations, Pergamon, London, 1957.

[3] D.S. Jones, The scattering of sound by a simple shear layer, Phil. Trans. Roy. Soc., London 284 (1977), 287-328.

[4] P.G. Drazin, and W.H. Reid, Hydrodynamic Stability, Cambridge, New York, 1982.

[5] Horace Lamb, Hydrodynamics, Dover, New York, 1932.

[6] D.V. Evans, The effect of surface tension on the waves produced by a heaving circular cylinder, Proc. Camb. Phil. Soc., 64 (1968), 833-847.

[7] W. Ewing, W. Jardetzky, and F. Press, Elastic Waves in Layered Media, McGraw-Hill, New York, 1957.

[8] G.M. Murphy, Ordinary Differential Equations and Their Solutions, D. Van Nostrand, Inc., London, 1960.

[9] P. A. Martin and A. G. Ramm, Inverse scattering for geophysical problems. III. On the velocity-inversion problems of acoustics, Proc. Roy. Soc., London, A 399, 153 (1985). 